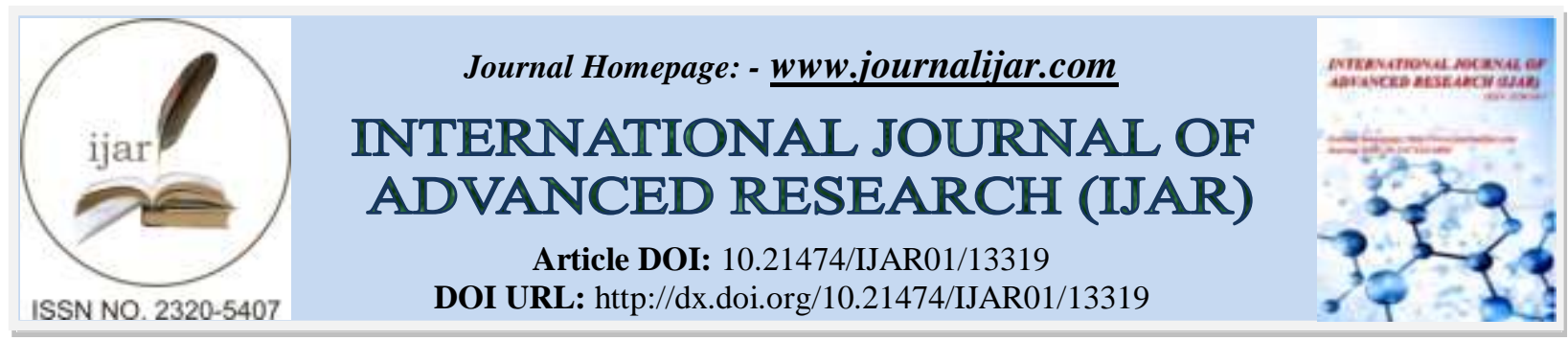

RESEARCH ARTICLE

\title{
A STUDY ON CORRELATION AND ASSOCIATION OF CHARACTERS OF EXACUM TETRAGONUM ROXB. IN NORTH KERALA, INDIA
}

\author{
K.S. Veena ${ }^{1}$, V.V. Radhakrishnan ${ }^{1}$ and K.V. Mohanan ${ }^{2}$ \\ 1. Genetics and Plant Breeding Division, Department of Botany, University of Calicut, Kerala - 673 635, India. \\ 2. Gregor Mendel Foundation, Calicut University (PO), Kerala - 673 635, India.
}

\section{Manuscript Info}

..........................

Manuscript History

Received: 25 June 2021

Final Accepted: 28 July 2021

Published: August 2021

Key words:-

Exacum Tetragonum, Interrelationship, Association, Correlation, Variability, Drastic Changes

\section{Abstract}

Western Ghats provide asylum to several endemic plants. Exacum tetragonum Roxb. is one of the important endemic herbs inhabiting the hilly tracts and midlands of Kerala, the state of India located towards the south-western side of the Western Ghats. The plant is used for treating fever, stomach disorders, gout, diabetics, and eye diseases. It is also found associated with antioxidant, anti-inflammatory, antihelminthic and anti-hyperglycemic activities. Correlation and association of characters of this important medicinal plant was analyzed based on the observations on thirteen salient morphological characters recorded from twenty three populations of the species distributed across Malappuram, Thrissur, Kozhikode and Palakkad Districts of Kerala State of India. All the characters studied such as plant height, number of leaves, number of flowers, number of inflorescences, leaf length, leaf breadth, peduncle length, pedicel length, internodal length, leaf area, mean stem girth, number of branches and fresh weight of plants showed statistically significant variations between the populations. Number of flowers showed significant positive correlation with the maximum number of characters (ten characters) whereas number of leaves showed significant positive correlation with the minimum number of characters (two characters). By factor analysis, two factors could be extracted and fresh weight of plant was found to be the lead character. Significant variability with regard to morphological characters indicates the strong genetic base of the plant species in the field. However, drastic changes in its habitat for various reasons can certainly cause severe threats to its continued existence and hence appropriate actions to conserve the natural habitats of this species are vital.

Copy Right, IJAR, 2021,. All rights reserved.

\section{Introduction:-}

Exacum tetragonum is a herbaceous medicinal plant with bicoloured petals and it comes under the family Gentianaceae. Its natural habitat is tropical moist grassy forests. The species is widespread in Indomalesia and reaches the extreme north of Australia (Yuan et al., 2005). About three to four decades ago, the plant was seen abundantly in different parts of Kerala. The plant is not amenable to propagation in gardens. The plant is known as kannanthali in Malayalam and Persian blue violet in English (Sreelatha et al., 2007). It is regarded as a promising 
anti-oxidant, thrombolytic and anti-inflammatory agent (Ashwini et al., 2015). It contains various phytochemicals such as phenols, saponins, flavonoids and alkaloids. Naga people have been using Exacum tetragonum against malaria fever since long (Rao, 1983). The plant is used as an antidiabetic herb in Kerala (Sreelatha et al., 2007). In Khao Kho District of Thailand the species is considered as a valuable medicinal herb (Chuakul, 2000). Roots of Exacum tetragonum contain a colouring matter and hence it is used in alcoholic drinks in Germany and Switzerland (Torfida, 1944). Whole plant is used as tonic and stomachic by Ambalabe rural community (Vinayaka et al., 2016). Decoction of leaves and bark of Exacum tetragonum is used by Angami Nagas for curing malarial fever (Rao, 1983). It is widely used for curing human ailments like diabetes, malaria, skin disorders, fungal diseases and inflammation (Marles and Farnsworth, 1995).

The species is seen limited to small pocket areas even in vast stretches of grassland. This unique feature accompanied by the drastic social changes has subjected the plant to the threat of extinction (Sreelatha et al., 2007). The forest ecosystems of Western Ghats are under severe threat due to a variety of reasons. Understanding the genetics and interrelationship of the quantitative characters of such plants is an important step towards developing strategies for conservation. Hence the present study has been carried out to analyze the correlation and association of characters of Exacum tetragonum based on thirteen morphological characters (Table 1).

\section{Materials and Methods:-}

Twenty three natural populations of the plant were located at different parts of Malappuram, Thrissur, Kozhikode and Palakkad Districts of Kerala State of India during August-November 2018. The populations were frequently visited to assess their growth and when the plants matured and flowered, twelve plants were collected at random from each population so as to represent intrapopulational variability appropriately. The collected plants were observed for thirteen quantitative morphological characters. Correlation of these characters was analyzed as suggested by Rangaswami (1995). Study of association of characters was carried out to group the characters based on their relationship and also to find out the lead characters in each group. Study of character association of Exacum tatragonum was carried out using principal component analysis with the help of the statistical software STATISTICA.

\section{Results and Discussion:-}

Most of the growth and yield characters of plants are polygenic in nature. Polygenic systems are complex systems in which alleles located at different loci contribute towards the expression of other related characters also. Hence different levels of interrelationships can be observed between polygenic quantitative characters. The extent of this relationship can be analyzed using correlation analysis. In the present study, plant height showed significant positive correlation with number of flowers, leaf length, peduncle length, pedicel length, internodal length, mean stem girth and fresh weight of plants; number of leaves showed significant positive correlation with leaf length and number of branches; number of flowers showed significant positive correlation with plant height, number of inflorescences, leaf length, peduncle length, pedicel length, intermodal length, leaf area, mean stem girth, number of branches and fresh weight of plants; number of inflorescences showed significant positive correlation with number of flowers, leaf length, peduncle length, pedicel length, internodal length, leaf area, mean stem girth and fresh weight of plants; leaf length showed significant positive correlation with plant height, number of flowers, number of inflorescences, leaf breadth, internodal length, leaf area, mean stem girth and fresh weight of plants; leaf breadth showed significant positive correlation with number of leaves, leaf length, leaf area and number of branches; peduncle length showed significant positive correlation with plant height, number of flowers, number of inflorescences, pedicel length, internodal length, mean stem girth and fresh weight of plants; pedicel length showed significant positive correlation with plant height, number of flowers, number of inflorescences, peduncle length, internodal length, mean stem girth and fresh weight of plants; internodal length showed significant positive correlation with plant height, number of flowers, leaf length, peduncle length, pedicel length, leaf area and fresh weight of plants; mean stem girth showed significant positive correlation with plant height, number of flowers, number of inflorescences, leaf length, peduncle length, pedicel length, leaf area and fresh weight of plants; leaf area showed significant positive correlation with number of flowers, number of inflorescences, leaf length, leaf breadth, internodal length, mean stem girth and fresh weight of plants; number of branches showed significant positive correlation with number of leaves, number of flowers and leaf breadth; fresh weight of plants showed significant positive correlation with plant height, number of flowers, number of inflorescences, leaf length, peduncle length, pedicel length, internodal length, leaf area and mean stem girth (Tables 2 and 3). Number of flowers showed significant positive correlation with the maximum number of characters (ten characters) whereas number of leaves 
showed significant positive correlation with the minimum number of characters (two characters). Characters having interrelationship with the maximum number of characters show high level of gene sharing. Analysis of such interrelationships has been already carried out in other economically important plant species by earlier workers like Sankaran et al. (1994) and Priya et al. (2012).

Factor analysis of thirteen morphological characters of Exacum tetragonum has been studied based on UPGMA method using STATISTICA software. Two factors were extracted from the data based on the relative contribution of the variable towards the variability of the species. Based on factor loading, plant height, number of flowers, numbers of inflorescences, leaf length, peduncle length, pedicel length, internodal length, leaf area, mean stem girth and fresh weight were grouped under factor 1 and number of leaves, leaf breadth and number of branches under factor 2 (Tables 4, 5 and 6).

It can be assumed that there is a sharing of common alleles between plant height, number of flowers, numbers of inflorescences, leaf length, peduncle length, pedicel length, intermodal length, leaf area, mean stem girth and fresh weight come under factor 1 and there is a sharing of common alleles between number of leaves, leaf breadth and number of branches under factor 2. In factor 1, fresh weight can be considered the lead character since it shows the maximum factor loading.

Grouping of variables can be used as a tool in selection programmes in future when commercialization of medicinal plant production may necessitate development of improved strains. Factor analysis has been used to group variables in different plants like rubber (Abraham et al., 2002), cardamom (Radhakrishnan et al., 2004) and chillies (Hrideek et al., 2006). Though considerably high field level variability is present in the plant species under study, steps should be taken to conserve its natural habitats from anthropogenic destruction and to augment its genetic base.

Table 1:- Growth and yield characters of Exacum tetragonum studied.

\begin{tabular}{|l|l|l|l|l|}
\hline Sl. No. & Character & Mean \pm SE & CD $(5 \%)$ & CV $(\%)$ \\
\hline 1 & Plant height $(\mathrm{cm})$ & $52.89 \pm 3.23$ & 9.25 & 30.99 \\
\hline 2 & Number of leaves & $21.63 \pm 1.61$ & 5.31 & 24.64 \\
\hline 3 & Number of flowers & $24.66 \pm 3.07$ & 9.09 & 41.36 \\
\hline 4 & Number of inflorescences & $4.96 \pm 0.55$ & 1.69 & 39.52 \\
\hline 5 & Leaf length $(\mathrm{cm})$ & $5.83 \pm 0.27$ & 0.78 & 19.90 \\
\hline 6 & Leaf breadth $(\mathrm{cm})$ & $1.45 \pm 0.08$ & 0.20 & 11.72 \\
\hline 7 & Peduncle length $(\mathrm{cm})$ & $3.02 \pm 0.27$ & 0.69 & 32.45 \\
\hline 8 & Pedicel length $(\mathrm{cm})$ & $1.15 \pm 0.13$ & 0.33 & 27.83 \\
\hline 9 & Internodal length $(\mathrm{cm})$ & $4.50 \pm 0.21$ & 0.65 & 25.56 \\
\hline 10 & Mean stem girth $(\mathrm{cm})$ & $1.51 \pm 0.08$ & 0.27 & 16.67 \\
\hline 11 & Leaf area $\left(\mathrm{cm}^{2}\right)$ & $748.90 \pm 62.99$ & 188.04 & 23.89 \\
\hline 12 & Number of branches & $1.61 \pm 0.04$ & 0.27 & 39.75 \\
\hline 13 & Fresh weight $(\mathrm{g})$ & $18.62 \pm 1.83$ & 5.41 & 44.58 \\
\hline
\end{tabular}

Table 2:- Exacum tetragonum - Correlation of characters.

\begin{tabular}{|c|c|c|c|c|c|c|c|c|c|c|c|c|c|}
\hline & $\begin{array}{l}\text { Pla } \\
\text { nt } \\
\text { heig } \\
\text { ht }\end{array}$ & $\begin{array}{l}\text { Numb } \\
\text { er of } \\
\text { leaves }\end{array}$ & $\begin{array}{l}\text { Numb } \\
\text { er of } \\
\text { flower } \\
\text { s }\end{array}$ & $\begin{array}{l}\text { Numb } \\
\text { er of } \\
\text { inflore } \\
\text { s } \\
\text { cences }\end{array}$ & $\begin{array}{l}\text { Lea } \\
\mathrm{f} \\
\text { len } \\
\text { gth }\end{array}$ & $\begin{array}{l}\text { Leaf } \\
\text { brea } \\
\text { dth }\end{array}$ & $\begin{array}{l}\text { Pedu } \\
\text { ncle } \\
\text { lengt } \\
\text { h }\end{array}$ & $\begin{array}{l}\text { Pedi } \\
\text { cel } \\
\text { lengt } \\
\text { h }\end{array}$ & $\begin{array}{l}\text { Intern } \\
\text { odal } \\
\text { lengt } \\
\mathrm{h}\end{array}$ & $\begin{array}{l}\text { Mean } \\
\text { stem } \\
\text { girth }\end{array}$ & $\begin{array}{l}\text { Lea } \\
\mathrm{f} \\
\text { are } \\
\mathrm{a}\end{array}$ & $\begin{array}{l}\text { Numbe } \\
\text { r of } \\
\text { branch } \\
\text { es }\end{array}$ & $\begin{array}{l}\text { Fres } \\
\text { h } \\
\text { wei } \\
\text { ght }\end{array}$ \\
\hline $\begin{array}{l}\text { Plant } \\
\text { height }\end{array}$ & 1 & & & & & & & & & & & & \\
\hline $\begin{array}{l}\text { Number } \\
\text { of leaves }\end{array}$ & $\begin{array}{l}0.2 \\
814 \\
77 \\
\end{array}$ & 1 & & & & & & & & & & & \\
\hline $\begin{array}{l}\text { Number } \\
\text { of } \\
\text { flowers }\end{array}$ & $\begin{array}{l}0.5 \\
354 \\
45^{*}\end{array}$ & $\begin{array}{l}- \\
0.106 \\
96\end{array}$ & 1 & & & & & & & & & & \\
\hline Number & 0.3 & - & 0.8374 & 1 & & & & & & & & & \\
\hline
\end{tabular}




\begin{tabular}{|c|c|c|c|c|c|c|c|c|c|c|c|c|c|}
\hline $\begin{array}{l}\text { of } \\
\text { infloresc } \\
\text { ences }\end{array}$ & $\begin{array}{l}330 \\
11\end{array}$ & $\begin{array}{l}0.186 \\
88\end{array}$ & $02 *$ & & & & & & & & & & \\
\hline $\begin{array}{l}\text { Leaf } \\
\text { length }\end{array}$ & $\begin{array}{l}0.3 \\
555 \\
8^{*}\end{array}$ & $\begin{array}{l}- \\
0.028 \\
61\end{array}$ & $\begin{array}{l}0.6291 \\
34 *\end{array}$ & $\begin{array}{l}0.6607 \\
51^{*}\end{array}$ & 1 & & & & & & & & \\
\hline $\begin{array}{l}\text { Leaf } \\
\text { breadth }\end{array}$ & $\begin{array}{l}0.2 \\
257 \\
17\end{array}$ & $\begin{array}{l}0.407 \\
299 *\end{array}$ & $\begin{array}{l}0.0545 \\
91\end{array}$ & $\begin{array}{l}0.1815 \\
34\end{array}$ & $\begin{array}{l}0.4 \\
879 \\
63^{*}\end{array}$ & 1 & & & & & & & \\
\hline $\begin{array}{l}\text { Peduncle } \\
\text { length }\end{array}$ & $\begin{array}{l}0.7 \\
146 \\
09 *\end{array}$ & $\begin{array}{l}0.078 \\
778\end{array}$ & $\begin{array}{l}0.6830 \\
15^{*}\end{array}$ & $\begin{array}{l}0.4630 \\
54 *\end{array}$ & $\begin{array}{l}0.2 \\
675 \\
29\end{array}$ & $\begin{array}{l}- \\
0.01 \\
948\end{array}$ & 1 & & & & & & \\
\hline $\begin{array}{l}\text { Pedicel } \\
\text { length }\end{array}$ & $\begin{array}{l}0.5 \\
982 \\
64 *\end{array}$ & $\begin{array}{l}0.206 \\
163\end{array}$ & $\begin{array}{l}0.5757 \\
55^{*}\end{array}$ & $\begin{array}{l}0.4779 \\
43^{*}\end{array}$ & $\begin{array}{l}0.1 \\
793 \\
42\end{array}$ & $\begin{array}{l}0.15 \\
654 \\
2\end{array}$ & $\begin{array}{l}0.839 \\
155^{*}\end{array}$ & 1 & & & & & \\
\hline $\begin{array}{l}\text { Internod } \\
\text { al length }\end{array}$ & $\begin{array}{l}0.8 \\
557 \\
57 *\end{array}$ & $\begin{array}{l}0.277 \\
335\end{array}$ & $\begin{array}{l}0.3553 \\
8^{*}\end{array}$ & $\begin{array}{l}0.2651 \\
53\end{array}$ & $\begin{array}{l}0.3 \\
843 \\
82 *\end{array}$ & $\begin{array}{l}0.32 \\
297 \\
8\end{array}$ & $\begin{array}{l}0.613 \\
53^{*}\end{array}$ & $\begin{array}{l}0.60 \\
8787 \\
*\end{array}$ & 1 & & & & \\
\hline $\begin{array}{l}\text { Mean } \\
\text { stem } \\
\text { girth }\end{array}$ & $\begin{array}{l}0.5 \\
438 \\
45^{*}\end{array}$ & $\begin{array}{l}0.208 \\
993\end{array}$ & $\begin{array}{l}0.7857 \\
84^{*}\end{array}$ & $\begin{array}{l}0.6206 \\
17^{*}\end{array}$ & $\begin{array}{l}0.4 \\
805 \\
24 *\end{array}$ & $\begin{array}{l}0.21 \\
902 \\
8\end{array}$ & $\begin{array}{l}0.596 \\
579 *\end{array}$ & $\begin{array}{l}0.68 \\
6809 \\
*\end{array}$ & $\begin{array}{l}0.347 \\
386\end{array}$ & 1 & & & \\
\hline $\begin{array}{l}\text { Leaf } \\
\text { area }\end{array}$ & $\begin{array}{l}0.3 \\
183 \\
17\end{array}$ & $\begin{array}{l}0.069 \\
076\end{array}$ & $\begin{array}{l}0.6248 \\
53 *\end{array}$ & $\begin{array}{l}0.6741 \\
56^{*}\end{array}$ & $\begin{array}{l}0.9 \\
488 \\
53 *\end{array}$ & $\begin{array}{l}0.65 \\
065 \\
4^{*}\end{array}$ & $\begin{array}{l}0.278 \\
076\end{array}$ & $\begin{array}{l}0.26 \\
0057\end{array}$ & $\begin{array}{l}0.355 \\
828^{*}\end{array}$ & $\begin{array}{l}0.528 \\
862 *\end{array}$ & 1 & & \\
\hline $\begin{array}{l}\text { Number } \\
\text { of } \\
\text { branches }\end{array}$ & $\begin{array}{l}- \\
0.2 \\
568 \\
5\end{array}$ & $\begin{array}{l}0.722 \\
18^{*}\end{array}$ & $\begin{array}{l}- \\
0.3625 \\
8^{*}\end{array}$ & $\begin{array}{l}- \\
0.2481 \\
3\end{array}$ & $\begin{array}{l}- \\
0.1 \\
257 \\
5\end{array}$ & $\begin{array}{l}0.42 \\
319 \\
5^{*}\end{array}$ & $\begin{array}{l}- \\
0.324 \\
41\end{array}$ & $\begin{array}{l}- \\
0.12 \\
482\end{array}$ & $\begin{array}{l}- \\
0.186 \\
06\end{array}$ & $\begin{array}{l}- \\
0.013 \\
44\end{array}$ & $\begin{array}{l}0.0 \\
188 \\
22\end{array}$ & 1 & \\
\hline $\begin{array}{l}\text { Fresh } \\
\text { weight }\end{array}$ & $\begin{array}{l}0.6 \\
843 \\
1^{*}\end{array}$ & $\begin{array}{l}0.176 \\
275\end{array}$ & $\begin{array}{l}0.8135 \\
38^{*}\end{array}$ & $\begin{array}{l}0.6543 \\
13 *\end{array}$ & $\begin{array}{l}0.6 \\
289 \\
43 *\end{array}$ & $\begin{array}{l}0.23 \\
835\end{array}$ & $\begin{array}{l}0.640 \\
205^{*}\end{array}$ & $\begin{array}{l}0.56 \\
1966 \\
*\end{array}$ & $\begin{array}{l}0.566 \\
55^{*}\end{array}$ & $\begin{array}{l}0.811 \\
468^{*}\end{array}$ & $\begin{array}{l}0.6 \\
171 \\
79^{*}\end{array}$ & $\begin{array}{l}0.1264 \\
9\end{array}$ & 1 \\
\hline
\end{tabular}

Table 3:- Exacum tetragonum - Correlation of characters - Characters correlated.

\begin{tabular}{|c|c|}
\hline Characters & Characters showing significant positive correlation \\
\hline Plant height & $\begin{array}{l}\text { Number of flowers, leaf length, peduncle length, pedicel length, } \\
\text { intermodal length, mean stem girth and fresh weight }\end{array}$ \\
\hline Number of leaves & Leaf length and number of branches \\
\hline Number of flowers & $\begin{array}{l}\text { Plant height, number of inflorescences, leaf length, peduncle length, } \\
\text { pedicel length, intermodal length, leaf area, mean stem girth, number } \\
\text { of branches and fresh weight }\end{array}$ \\
\hline Number of inflorescences & $\begin{array}{l}\text { Number of flowers, leaf length, peduncle length, pedicel length, } \\
\text { intermodal length, leaf area, mean stem girth and fresh weight }\end{array}$ \\
\hline Leaf length & $\begin{array}{l}\text { Plant height, number of flowers, number of inflorescences, leaf } \\
\text { breadth, intermodal length, leaf area, mean stem girth and fresh weight }\end{array}$ \\
\hline Leaf breadth & Number of leaves, leaf length, leaf area and number of branches \\
\hline Peduncle length & $\begin{array}{l}\text { Plant height, number of flowers, number of inflorescences, pedicel } \\
\text { length, intermodal length, mean stem girth and fresh weight }\end{array}$ \\
\hline Pedicel length & $\begin{array}{l}\text { Plant height, number of flowers, number of inflorescences, peduncle } \\
\text { length, intermodal length, mean stem girth and fresh weight }\end{array}$ \\
\hline Internodal length & $\begin{array}{l}\text { Plant height, number of flowers, leaf length, peduncle length, pedicel } \\
\text { length, leaf area and fresh weight }\end{array}$ \\
\hline Mean stem girth & $\begin{array}{l}\text { Plant height, number of flowers, number of inflorescences, leaf } \\
\text { length, peduncle length, pedicel length, leaf area and fresh weight }\end{array}$ \\
\hline Leaf area & $\begin{array}{l}\text { Number of flowers, number of inflorescences, leaf length, leaf } \\
\text { breadth, intermodal length, mean stem girth and fresh weight }\end{array}$ \\
\hline
\end{tabular}




\begin{tabular}{|l|l|}
\hline Number of branches & Number of leaves, number of flowers and leaf breadth \\
\hline Fresh weight & $\begin{array}{l}\text { Plant height, number of flowers, number of inflorescences, leaf length, } \\
\text { peduncle length, pedicel length, intermodal length, leaf area and mean } \\
\text { stem girth. }\end{array}$ \\
\hline
\end{tabular}

Table 4:- Factor analysis of thirteen characters of Exacum tetragonum- Eigen values.

\begin{tabular}{|c|l|l|l|l|}
\hline Factor & \multicolumn{1}{|c|}{ Eigen value } & $\begin{array}{c}\text { \% of total } \\
\text { variance }\end{array}$ & $\begin{array}{c}\text { Cumulative } \\
\text { Eigen value }\end{array}$ & Cumulative \% of total variance \\
\hline 1 & 6.275553 & 48.27348 & 6.27555 & 48.27348 \\
\hline 2 & 2.277164 & 17.51665 & 8.55272 & 65.79013 \\
\hline
\end{tabular}

Table 5:-Factor analysis of thirteen characters of Exacum tetragonum- Factor Loadings.

\begin{tabular}{|l|l|l|}
\hline Characters & Factor I & Factor II \\
\hline Plant height & $\mathbf{0 . 7 6 3 7 3 9}$ & -0.002627 \\
\hline Number of leaves & 0.138896 & $\mathbf{- 0 . 7 9 9 3 3 0}$ \\
\hline Number of flowers & $\mathbf{0 . 8 7 5 0 2 0}$ & 0.285939 \\
\hline Number of inflorescences & $\mathbf{0 . 7 6 5 4 6 9}$ & 0.193441 \\
\hline Leaf length & $\mathbf{0 . 7 1 7 0 4 6}$ & -0.160003 \\
\hline Leaf breadth & 0.354235 & $\mathbf{- 0 . 7 5 0 7 6 5}$ \\
\hline Peduncle length & $\mathbf{0 . 7 7 2 2 7 3}$ & 0.268638 \\
\hline Pedicel length & $\mathbf{0 . 7 3 8 0 3 8}$ & 0.071851 \\
\hline Internodal length & $\mathbf{0 . 6 8 5 3 1 0}$ & -0.108963 \\
\hline Mean stem girth & $\mathbf{0 . 8 2 5 3 9 2}$ & -0.044414 \\
\hline Leaf area & $\mathbf{0 . 7 3 2 4 5 5}$ & -0.301032 \\
\hline Number of branches & -0.213148 & $\mathbf{- 0 . 8 6 4 6 5 2}$ \\
\hline Fresh weight & $\mathbf{0 . 8 9 8 7 6 3}$ & -0.019508 \\
\hline
\end{tabular}

Table 6:- Factor analysis - Characters associated with factors.

\begin{tabular}{|l|l|}
\hline Factor & Variables \\
\hline 1 & $\begin{array}{l}\text { Plant height, number of flowers, number of } \\
\text { inflorescences, leaf length, peduncle length, pedicel } \\
\text { length, intermodal length, leaf area, mean stem girth, } \\
\text { fresh weight }\end{array}$ \\
\hline 2 & Number of leaves, leaf breadth, number of branches \\
\hline
\end{tabular}

\section{References:-}

1. Abraham, S.T., Panikker, A.O.N., George, P.J., Nair, R.B., Raghu C.P. \& Annamma V. (2002). Factor analysis in certain wild genotype of Hevea brasiliensis. In: Sreedharan, K., Vinodkumar, P.K., Jayaram \& Basavaraj, M.C. (Eds.) Proceeding of Placrosym XV. Central Coffee Research Institute, Chickmagalore, Karnataka, India. p. 14-19.

2. Ashwini, A.M., Puttarudrappa, L., Ravi, B.V. \& Majumdar, M. (2015). GC-MS analysis, evaluation of phytochemicals, anti-oxidant, thrombolytic and anti-inflammatory activities of Exacum bicolor. Bangladesh J. Pharmacol., 10: 745-752.

3. Chuakul, W. (2000). Medicinal plants in Khao Kho district, Phetchabun province, Thailand. Pharmaceutical Biology, 38(1): 61-68.

4. Hrideek, T.K., Priya, P.M., Kuruvilla, K.M., Madhusoodanan, K.J. \& Thomas, J. (2006). Factor analysis in exotic chillies. Indian Journal of Agricultural Research, 40(4): 277-281.

5. Marles, R.J. \& Farnsworth, N.R. (1995). Antidiabetic plants and their active constituents. Phytomedicine, 2: 137-139.

6. Priya, P.M., Kuruvilla, K.M. \& Madhusoodanan, K.J. (2002). Yield prediction in Vanilla (Vanilla planifolia Andrews). In: Sreedharan, K., Kumar, P.K.V., Jayaram \& Basavaraj M.C. (Eds.) Proc. Placrosym XV. Central Coffee Research Institute, Chickmagalore, Karnataka, India. p. 414-415.

7. Radhakrishnan, V.V., Madhusoodanan, K.J., Priya, P.M., Mohanan, K.V., Kuruvilla, K.M. \& Thomas, J. (2004). Evaluation of promising lines of cardamom for yield and quality. Journal of Plantation Crops, 32(Suppl.): 30-32. 
8. Rangaswamy, R. (1995). A Text Book of Agricultural Statistics. New Age International Publishers Ltd., New Delhi, India. p.495.

9. Rao, M.R.R. (1983). Ethnobotanical studies in Nagaland medicinal plants used by the Angami Nagas. J. Econ. Tax. Bot., 4: 167-172.

10. Sankaran, P.G., Kuruvilla, K.M. \& Madhusoodanan, K.J. (1994). Correlation between biometrical characters in Vanilla (Vanilla planifolia Andrews). Journal of Spices and Aromatic Crops, 3(2): 155-157.

11. Sreelatha, U., Baburaj, T.S., Kutty, C.N., Nazeem, P.A. \& Bhaskar, J. (2007). Cultivation prospects of Exacum bicolor Roxb., an endangered, ornamental and anti-diabetic herb. Indian Journal of Natural Products and Resource, 6(5): 402-404.

12. Torfrida, (1944). Nurseries of heaven wild flowers of India. Thacker \& Co. Ltd, Bombay. p.322.

13. Vinayaka, K.S., Ashwini, H.S., Prashith, K.T.R. \& Krishanamurthy, Y.L. (2016). Traditional utilization and phytochemical analysis of medicinal herb Exacum Linn. from central Western Ghats. Asian Pacific Journal of Health Sciences, 3(1): 161-164.

14. Yuan, Y.M., Wohlhauser, S., Möller, M., Klackenberg, J., Callmander, M.W. \& Küpfer, P. (2005). Phylogeny and biogeography of Exacum (Gentianaceae): A disjunctive distribution in the Indian Ocean Basin resulted from long distance dispersal and extensive radiation. Syst. Biol., 54: 21-34. 